\title{
Reduced Matrix Elements for Symmetry-Constructed Systems ${ }^{\dagger}$
}

\author{
Marion Lawrence Ellzey, Jr
}

Department of Chemistry, The University of Texas at El Paso, 500 West University, El Paso, Texas 79968, USA

(E-mail: lellzey@utep.edu)

RECEIVED JUNE 16, 2013; REVISED JULY 29, 2013; ACCEPTED JULY 30, 2013

\begin{abstract}
Eigenvalue problems involving symmetry, such as the Schrödinger equation when the Hamiltonian commutes with a group, can generally be reduced in size using group theoretical techniques such as the Wigner-Eckart theorem. The key step is calculation of the reduced matrix elements followed by eigenvalue determination by the secular equation. For finite groups it is usual to obtain reduced matrices by transformation to the symmetry adapted basis. Direct determination of reduced matrix elements by some means would be computationally more efficient with better precision.It is shown here that this direct determination is possible to some extent for symmetry-constructed systems such as symmetry-generated molecules. A simple illustration is given using the Hûckel treatment of the cyclopropenyl radical (doi: $10.5562 / \mathrm{cca} 2307)$
\end{abstract}

Keywords: symmetry methods, Wigner-Eckart theorem, group representations

\section{INTRODUCTION}

A symmetry-constructed system is a sum of terms each of which is a transformation of some basic unit by an element of the symmetry group. ${ }^{1,2}$ Let the Hamiltonian operator of term $a$ be $H_{a}$ which is obtained from the basic unit $H_{0}$ by the group element $G_{a}$ according to:

$$
H_{a}=G_{a} H_{0} G_{\bar{a}}
$$

Then the total Hamiltonian, $H_{\text {total }}$, which must commute with the symmetry group $\boldsymbol{G}$, is:

$$
H_{\text {total }}=\sum_{a=1}^{g} c_{a} G_{a} H_{0} G_{\bar{a}}
$$

where $g$ is the order of $\boldsymbol{G}$. In general, none of the parts, $H_{a}$, including $H_{0}$, commute with $\boldsymbol{G}$. Note that $G_{\bar{a}}$ indicates the inverse of $G_{a}$ and $G_{1}$ is the identity. The coefficients $c_{a}$ are scalars.

A particular choice for these coefficients might be:

$$
c_{a}=\left\{\begin{array}{l}
1, a=1, \ldots, n \\
0, a \succ n \leq g
\end{array}\right.
$$

so that: ${ }^{\text {(a) }}$

$$
H_{\text {total }}=\sum_{a=1}^{n} G_{a} H_{0} G_{\bar{a}}
$$

which is a symmetry-generation expression. ${ }^{3}$ Symmetry-generation in which all coefficents are one or zero is a special case of symmetry-construction.

Symmetry-construction is not unique. For a given $H_{\text {total }}$ there can be different possible choices of $H_{0}$ as well as sets of $c_{a}{ }^{3}$

\section{REDUCED MATRIX ELEMENTS OF SYMMETRY-CONSTRUCTED SYSTEMS}

Reduced matrices are generated over symmetry-adapted bases. Using the notation of $\mathrm{Weyl}^{4}$ a symmetry-adapted basis is written:

$$
\{|\omega ; \rho \alpha r\rangle, r=1, . ., f(\alpha) ; \alpha=1, . ., M ; \rho=1, . ., f(\omega ; \alpha)\}
$$

where $\alpha$ is one of the $M f(\alpha)$-dimensional irreducible representations of the group and $r$ indexes the columns. The index $\rho$ distinguishes repeated irreducible representations where the number of repetitions is given by the usual character formula:

$$
f(\omega ; \alpha)=\frac{1}{g} \sum_{\sigma=1}^{M} n_{\sigma} \chi_{\bar{\sigma}}^{\alpha} \chi_{\sigma}^{\omega}
$$

Here, $\chi_{\sigma}^{\omega}$ is the character of the $\sigma^{\text {th }}$ class in the reducible basis $\mathrm{B}(\omega)$ of the defining space $\mathrm{V}(\omega), \chi_{\bar{\sigma}}^{\alpha}$ is the

\footnotetext{
$\dagger^{\dagger}$ Dedicated to Professor Douglas Jay Klein on the occasion of his $70^{\text {th }}$ birthday.

(a) In Reference (1), the Equation (4) is written as $H=\sum_{a}^{n} G_{a}^{-1} H_{R} G_{a}$
} 
character of the inverse of the $\sigma^{\text {th }}$ class in the $\alpha$ irreducible representation of $\boldsymbol{G}$. The order of $\boldsymbol{G}$ is $g$ and $M$ is the number of classes of $\boldsymbol{G}$. A symmetry-adapted basis makes explicit the decomposition into irreducible invariant subspaces of $\boldsymbol{G}$ :

$$
G_{\alpha}|\omega ; \rho \alpha r\rangle=\sum_{r^{\prime}=1}^{f(\alpha)}\left[G_{a}\right]_{r^{\prime} r}^{\rho \alpha}\left|\omega ; \rho \alpha r^{\prime}\right\rangle
$$

By definition, as given in Reference (1), in a suitably-conditioned symmetry-adapted basis all repeated irreducible representations are identical, not just equivalent:

$$
\left[G_{a}\right]^{\rho \alpha}=\left[G_{a}\right], \rho=1, \ldots, f(\omega ; \alpha)
$$

An algorithm for automatically generating a symmetry-adapted basis that is suitably-conditioned is given in Reference (1).

Matrix elements of operators that commute with the group $\boldsymbol{G}$, such as the total Hamiltonian, $H_{\text {total }}$, over the symmetry-adapted basis are zero unless $\alpha=\alpha^{\prime}$ and $r=r^{\prime}$ and are independent of $r$ :

$$
\begin{aligned}
& \left\langle\omega ; \rho \alpha r\left|H_{\text {total }}\right| \omega ; \rho^{\prime} \alpha^{\prime} r^{\prime}\right\rangle= \\
& =\delta\left(r, r^{\prime}\right) \delta\left(\alpha, \alpha^{\prime}\right)\left\langle\omega ; \rho \alpha\left\|H_{\text {total }}\right\| \omega ; \rho^{\prime} \alpha\right\rangle
\end{aligned}
$$

The bracket on the right is termed the reduced matrix element since it is independent of $r$. Equation (9) is a form of the Wigner-Eckart Theorem.

The block structure of the matrix of $H_{\text {total }}$ depends on the ordering of the basis elements. Grouping together the $r$ with the same $\rho$ as in Equation (7) indexes the blocks according to $\rho$ : $\left[H_{\text {total }}\right]^{\rho, \rho^{\prime} ; \alpha}$. Each block is a square scalar matrix with reduced matrix elements repeated $f(\alpha)$ times down the diagonal.

The matrix form of Equation (2) on a suitablyconditioned symmetry-adapted basis is:

$$
\begin{aligned}
{\left[H_{\text {total }}\right]^{\rho, \rho^{\prime} ; \alpha} } & =\sum_{a=1}^{g} c_{a}\left[G_{a}\right]^{\rho \alpha}\left[H_{0}\right]^{\rho, \rho^{\prime} ; \alpha}\left[G_{\bar{a}}\right]^{\rho^{\prime} \alpha} \\
& =\sum_{a=1}^{g} c_{a}\left[G_{a}\right]^{\alpha}\left[H_{0}\right]^{\rho, \rho^{\prime} ; \alpha}\left[G_{\bar{a}}\right]^{\alpha}
\end{aligned}
$$

Each term on the right of Equation (10) is an equivalence transformation of $\left[H_{0}\right]^{\rho, \rho^{\prime} ; \alpha}$. Taking the trace of both sides yields:

$$
\begin{aligned}
& f(\alpha)\left\langle\omega ; \rho \alpha\left\|H_{\text {total }}\right\| \omega ; \rho^{\prime} \alpha\right\rangle= \\
& =\left(\sum_{r=1}^{f(\alpha)}\left\langle\omega ; \rho \alpha r\left|H_{0}\right| \omega ; \rho^{\prime} \alpha r\right\rangle\right)\left(\sum_{a=1}^{g} c_{a}\right)
\end{aligned}
$$

Dividing by $f(\alpha)$ gives for the reduced matrix element:

$$
\begin{aligned}
& \left\langle\omega ; \rho \alpha \| H_{\text {total }}|| \omega ; \rho^{\prime} \alpha\right\rangle= \\
& =\left(\sum_{r=1}^{f(\alpha)}\left\langle\omega ; \rho \alpha r\left|H_{0}\right| \omega ; \rho^{\prime} \alpha r\right\rangle\right)\left(\frac{1}{f(\alpha)} \sum_{a=1}^{g} c_{a}\right)
\end{aligned}
$$

The first factor on the right is the trace of $\left[H_{0}\right]^{\rho, \rho^{\prime} ; \alpha}$. This expression gives the reduced matrix element directly in terms of the matrix elements of $H_{0}$ on the symmetry adapted basis.

\section{EXAMPLE}

A simple example illustrates the application of this method. The Hückel Hamiltonian of the cyclopropenyl radical can be symmetry-generated as:

$$
\left[H_{\text {total }}\right]=[E]\left[H_{0}\right][E]+\left[C_{3}\right]\left[H_{0}\right]\left[C_{3}^{2}\right]+\left[C_{3}^{2}\right]\left[H_{0}\right]\left[C_{3}\right]
$$

One choice of $\left[H_{0}\right]$ is:

$$
\left[H_{0}\right]=\left[\begin{array}{lll}
\alpha & \beta & 0 \\
\beta & 0 & 0 \\
0 & 0 & 0
\end{array}\right]
$$

so that:

$$
\begin{aligned}
{\left[\begin{array}{lll}
\alpha & \beta & \beta \\
\beta & \alpha & \beta \\
\beta & \beta & \alpha
\end{array}\right] } & =\left[\begin{array}{lll}
\alpha & \beta & 0 \\
\beta & 0 & 0 \\
0 & 0 & 0
\end{array}\right]+ \\
& +\left[\begin{array}{lll}
0 & 0 & 1 \\
1 & 0 & 0 \\
0 & 1 & 0
\end{array}\right]\left[\begin{array}{lll}
\alpha & \beta & 0 \\
\beta & 0 & 0 \\
0 & 0 & 0
\end{array}\right]\left[\begin{array}{lll}
0 & 1 & 0 \\
0 & 0 & 1 \\
1 & 0 & 0
\end{array}\right]+(15) \\
+ & {\left[\begin{array}{lll}
0 & 1 & 0 \\
0 & 0 & 1 \\
1 & 0 & 0
\end{array}\right]\left[\begin{array}{lll}
\alpha & \beta & 0 \\
\beta & 0 & 0 \\
0 & 0 & 0
\end{array}\right]\left[\begin{array}{lll}
0 & 0 & 1 \\
1 & 0 & 0 \\
0 & 1 & 0
\end{array}\right] }
\end{aligned}
$$

Another basic unit which gives the same result is:

$$
\left[H_{0}\right]=\left[\begin{array}{ccc}
\alpha / 2 & \beta & 0 \\
\beta & \alpha / 2 & 0 \\
0 & 0 & 0
\end{array}\right]
$$

The symmetry group may be taken to be either $\boldsymbol{C}_{3}$, $\boldsymbol{C}_{3 \mathrm{v}}, \boldsymbol{D}_{3}$ or $\boldsymbol{D}_{3 \mathrm{~h}}$. The group $\boldsymbol{C}_{3 \mathrm{v}}, g=6$, is chosen here for its simplicity and real irreducible representations. The coefficients in Equation (2) are one for $E, C_{3}, C_{3}^{2}$ and zero for remaining group elements. So:

$$
\sum_{a=1}^{g} c_{a}=3
$$


At this point there is no general algorithm for determining an $H_{0}$ and $c_{a}$ for a particular $H_{\text {total }}$.

The symmetry-adapted basis is:

$$
\left\{\left|\pi ; A_{1} 1\right\rangle,|\pi ; E 1\rangle,|\pi ; E 2\rangle\right\}
$$

with reduced matrix elements equal to Hückel eigenvalues:

$$
\begin{gathered}
\left\langle\pi ; A_{1}\left\|H_{\text {total }}\right\| \pi ; A_{1}\right\rangle=\alpha+2 \beta \\
\left\langle\pi ; E\left\|H_{\text {total }}\right\| \pi ; E\right\rangle=\alpha-\beta
\end{gathered}
$$

A choice for the symmetry-adapted basis is:

$$
\begin{aligned}
& \left|\pi ; A_{1} 1\right\rangle=\frac{1}{\sqrt{3}}\left(\left|\pi_{1}\right\rangle+\left|\pi_{2}\right\rangle+\left|\pi_{3}\right\rangle\right) \\
& |\pi ; E 1\rangle=\frac{1}{\sqrt{2}}\left(\left|\pi_{2}\right\rangle-\left|\pi_{3}\right\rangle\right) \\
& |\pi ; E 2\rangle=\frac{1}{\sqrt{6}}\left(2\left|\pi_{1}\right\rangle-\left|\pi_{2}\right\rangle-\left|\pi_{3}\right\rangle\right)
\end{aligned}
$$

Transforming the first $\left[H_{0}\right]$ gives:

$$
\begin{aligned}
& {\left[\begin{array}{ccc}
1 / \sqrt{3} & 1 / \sqrt{3} & 1 / \sqrt{3} \\
0 & 1 / \sqrt{2} & -1 / \sqrt{2} \\
2 / \sqrt{6} & -1 / \sqrt{6} & -1 / \sqrt{6}
\end{array}\right]\left[\begin{array}{ccc}
\alpha & \beta & 0 \\
\beta & 0 & 0 \\
0 & 0 & 0
\end{array}\right]\left[\begin{array}{ccc}
1 / \sqrt{3} & 0 & 2 / \sqrt{6} \\
1 / \sqrt{3} & 1 / \sqrt{2} & -1 / \sqrt{6} \\
1 / \sqrt{3} & -1 / \sqrt{2} & -1 / \sqrt{6}
\end{array}\right]=} \\
&=\left[\begin{array}{ccc}
\frac{1}{3}(\alpha+2 \beta) & \frac{1}{\sqrt{6}} \beta & \frac{1}{3 \sqrt{2}}(2 \alpha+\beta) \\
\frac{1}{\sqrt{6}} \beta & 0 & \frac{1}{\sqrt{3}} \beta \\
\frac{1}{3 \sqrt{2}}(2 \alpha+\beta) & \frac{1}{\sqrt{3}} \beta & \frac{2}{3}(\alpha-\beta)
\end{array}\right]
\end{aligned}
$$

The $A_{1}$ block is the upper left element and the $\mathrm{E}$ block is the lower right $2 \times 2$ unit. Using Equation (12) gives:

$$
\begin{aligned}
\left\langle\pi ; A_{1}\|H\| \pi ; A_{1}\right\rangle & =3\left\langle\pi ; A_{1} 1\left|H_{0}\right| \pi ; A_{1} 1\right\rangle \\
& =\alpha+2 \beta
\end{aligned}
$$

and

$$
\begin{aligned}
\langle\pi ; E\|H\| \pi ; E\rangle & =\frac{3}{2}\left(\left\langle\pi ; E 1\left|H_{0}\right| \pi ; E 1\right\rangle+\left\langle\pi ; E 2\left|H_{0}\right| \pi ; E 2\right\rangle\right) \\
& =\frac{3}{2}\left(0+\frac{2}{3}(\alpha-\beta)\right) \\
& =\alpha-\beta
\end{aligned}
$$

The other choice for $\left[H_{0}\right]$ in Equation (16) gives a different matrix but the same traces.

\section{CONCLUSION}

The usefulness of this method depends on the size and complexity of the application. It clearly has some interesting possibilities particularly for large molecules. One application not considered here is symmetry averaging where a high symmetry system is obtained from a low symmetry system by a process similar to symmetrygeneration. In this case, the transformations are performed on the whole system and not just a subunit. Possibilities for general symmetry-construction in which the coefficients in Equation (2) are other than zero or one remain to be explored.

\section{REFERENCES}

1. M. L. Ellzey, Jr., J. Chem. Inf. Comput. Sci. 43 (2003) 178-181.

2. M. L. Ellzey, Jr. and D. Villagran, J. Chem. Inf. Comput. Sci. 43 (2003) 1763-1770.

3. M. L. Ellzey, Jr., J. Comput. Chem. 28 (2007) 811-817.

4. H. Weyl, The Theory of Groups and Quantum Mechanics, Dover, New York, 1931 (H.P. Robertson, trans.). 